\title{
Análise de Métodos para Avaliar Dutos com Dano Mossa e Sulco
}

\author{
(Analysis of Methods to Assess Pipelines with Dent and Gouge Damage)
}

\author{
Marco Aurélio Oliveira Lima ${ }^{1}$, Sérgio Rodrigues Barra², José Luiz da França Freire ${ }^{3}$ \\ ${ }^{1}$ Det Norske Veritas, Energy Solutions South America, Salvador, Bahia, Brasil (marco.aurelio.lima@dnv.com) \\ ${ }^{2}$ Faculdade de Tecnologia SENAI Cimatec, Salvador,Bahia,Brasil (barra@cimatec.fieb.org.br) \\ ${ }^{3}$ PUC-Rio, Rio de Janeiro, Rio de Janeiro, Brasil (jlfreire@puc-rio.br)
}

\begin{abstract}
Resumo
A 'interferência externa', também conhecida como 'dano de terceira parte', se destaca como uma das principais causas de falhas em dutos onshore. Interferência externa pode causar, dentre outros, danos identificados como mossas, sulcos ou ambos, e também perfurações, conhecidos como 'dano mecânico'. Na literatura técnica existem alguns métodos analíticos semi-empíricos que se propoem a avaliar a adequação ao uso de dutos com dano mossa e sulco. Como as equações sugeridas por estes métodos diferem entre si, paira a dúvida sobre "qual" método daria um resultado mais exato quando aplicado. Sendo assim, este trabalho apresenta os resultados de uma análise técnica comparativa realizada para alguns destes métodos atualmente disponíveis, no tocante à fundamentação teórica, disponibilidade dos dados requeridos e exatidão em prever a tensão nominal causada pela pressão de falha. Os resultados obtidos mostram que existe uma grande similaridade entre os métodos estudados, que todos são, essencialmente, baseados na mecânica da fratura clássica, e que o 'método da fratura mossa e sulco semi-empírico original' desenvolvido pela British Gas se destaca como o 'mais recomendado' para avaliar a adequação ao uso de dutos com dano tipo 'mossa e sulco'.
\end{abstract}

Palavras-chave: Duto Onshore; Interferência Externa; Dano Mossa e Sulco; Avaliação de Adequação ao Uso.

Abstract: The 'external interference', also known as 'third party damage', stands out as one of the main causes of failures in onshore pipelines. External interference can cause, among others, damage identified as dents, gouges or both, and punctures, also known as 'mechanical damage'. In technical literature there are some semi-empirical analytical methods that aim to assess the fitness for purpose of pipelines with dent and gouge damage. As the equations suggested by these methods differ, the question hangs on "what" method would give a more accurate result when applied. Therefore, this paper presents the results of a comparative technique analysis for some of these methods currently available, concerning the theoretical basis, availability of required data and accuracy in predicting the nominal stress caused by the failure pressure. The results show that there is great similarity between these methods showed that all are essentially based on classical fracture mechanics, and that the 'original semi-empirical dent and gouge fracture method' developed by British Gas stands out as the "most recommended” to assess the fitness for purpose of pipelines with 'dent and gouge' damage type.

Key-words: Onshore Pipeline; External Interference; Damage Dent and Gouge; Fitness-For-Purpose Assessment.

\section{Introdução}

Dentro do segmento industrial de óleo e gás, dutos onshore (em terra) se destacam como um importante meio de transporte e distribuição de diversos produtos, seja na forma líquida (oleodutos) ou na forma gasosa (gasodutos).

Os produtos e substâncias normalmente transportados pelos dutos onshore se destacam pela suas características perigosas intrínsecas, tais como inflamabilidade e toxicidade. Estas características representam situações de risco para as pessoas, para as propriedades (máquinas, equipamentos, instalações prediais etc) e para o meio ambiente em que se encontram presentes, de forma fixa ou eventual, ao longo dos trajetos dos

(Recebido em 09/05/2010; Texto final em 11/11/2010). dutos.

Os riscos oferecidos às pessoas, às propriedades e ao meio ambiente, decorrentes da operação do duto com substâncias perigosas, necessitam então ser analisados e gerenciados. Uma das maneiras de gerenciar o risco, que pode, tradicionalmente, ser expresso conforme a Equação 1, é atuar na diminuição da frequência de falhas que ocasionam a liberação do produto perigoso, por meio de vazamento ou ruptura do duto.

Risco $=$ Frequência de Falha $x$ Consequência da Falha

A 'interferência externa' destaca-se, frequentemente, como a causa dominante de falhas em dutos onshore [1-6], podendo gerar nos mesmos danos tipo mossa, sulco, mossa e sulco, ou perfurações. Como exemplo, pode-se observar na Figura 1 que a interferência externa é a principal causa inicial de incidentes (liberação de gás não intencional) em dutos onshore de transporte de gás na Europa, correspondendo a aproximadamente $50 \%$ de 
todos os incidentes registrados pelo "European Gas Pipeline Incident Data Group" (EGIG) [5].

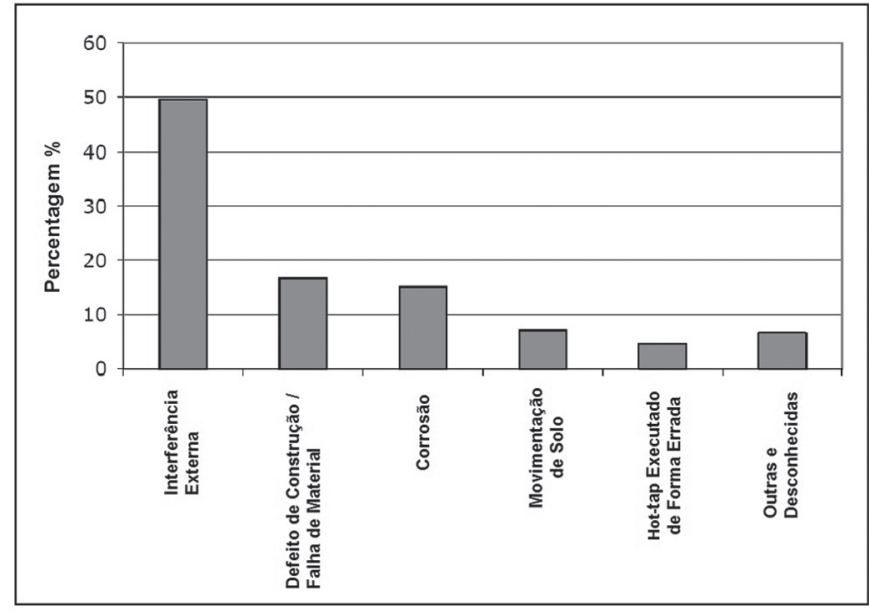

Figura 1 - Distribuição do percentual de incidentes, por tipo de causa, em dutos de gás na Europa [5].

Por conseguinte, conforme reportado em Seevam et al. [6], existe uma pressão sobre a indústria de dutos para entender o comportamento do dano mossa e sulco, em virtude de:

- Grande potencial para gerar danos às pessoas, às instalações e ao meio ambiente;

- Aumento do uso de técnicas de inspeção em dutos que estão apresentando tal dano;

- Rápida adoção de análise quantitativa de risco (AQR) ao redor do mundo.

Sendo assim, na área de dutos, esforços ao redor do mundo têm focado na necessidade de pesquisa para entendimento de dano mecânico, tal como o dano mossa e sulco. Nos Estados Unidos, a maioria do trabalho experimental para este tipo de dano tem sido conduzida pelo "Battelle Memorial Institute" e "Stress Engineering Services, Inc", com fomento da "American Gas Association" (AGA) e do "American Petroleum Institute" (API). Na Europa, testes têm sido conduzidos primariamente pela "British Gas" e "Gaz de France" com financiamento do "European Pipeline Research Group" (EPRG) [1]. No Brasil, observa-se um reduzido número de pesquisas e artigos técnicos focados na análise de dano mecânico em dutos, muitos deles desenvolvidos pelo CENPES (Petrobras) e pelo laboratório de fotomecânica da PUC-Rio.

Em função destas pesquisas, diversos métodos analíticos semi-empíricos que se propõem a avaliar a adequação ao uso de dutos com dano mossa e sulco têm sido publicados. Como as equações sugeridas por estes métodos diferem entre si, paira a dúvida sobre "qual" método daria um resultado mais exato, e deveria então ser aplicado. Diante deste fato, foi então decidido fazer uma análise técnica comparativa para alguns destes métodos atualmente disponíveis, no tocante à fundamentação teórica, disponibilidade dos dados requeridos e exatidão em prever a tensão de falha. Os resultados desta análise encontramse apresentados neste trabalho, onde evidencia-se que existe uma grande similaridade entre os métodos estudados, que todos são, essencialmente, baseados na mecânica da fratura clássica, e que o 'método da fratura mossa e sulco semi-empírico original', desenvolvido pela British Gas, se destaca como o 'mais recomendado' para avaliar a adequação ao uso de dutos com dano tipo 'mossa e sulco'.

\section{Apresentação de Parâmetros Relacionados ao Dano Mossa e Sulco}

\subsection{Interferência Externa}

A interferência externa, também conhecida como 'dano de terceira parte', é geralmente considerada como sendo o dano que ocorre ao duto quando escavação mecânica, perfuração, ou colisão de um equipamento/ferramenta sobre a parede de um duto enterrado gera arranhões, abrasões, sulcos, furos e/ou mossas na mesma. Dutos não enterrados (aparentes ou aéreos) podem também ser danificados de maneira similar devido a impactos de veículos, projéteis ou atos de vandalismo [7].

\subsection{Definição do Dano Mossa e Sulco}

A mossa é definida como uma depressão causada pelo contato de um corpo estranho (conhecido como 'indentador') contra o tubo do duto, resultando em deformação plástica da parede do tubo [9]. De forma mais abrangente, o API 579-1 / ASME FFS-1 [8] define mossa como sendo um desvio para dentro ou para fora da seção transversal de uma parte qualquer de um casco de geometria ideal.

Por sua vez, o sulco é definido como um dano de superfície causado pelo contato de um corpo estranho contra o duto com remoção de material do tubo, resultando em um defeito de perda de metal $[8,9]$.

A combinação dos danos mossa e sulco, também denominada de dano 'mossa e sulco', encontra-se ilustrada na Figura 2. Na mesma podem ser observadas, ilustrativamente, as dimensões que caracterizam o dano mossa e sulco.

\subsection{Caracterização da Falha Decorrente do Dano Mossa e Sulco}

Mayfield et al. [10] e Cosham [11] descrevem que o sulco pode conter micro-trincas, formadas durante o processo de geração do mesmo (arrancamento de metal) ou que tenham sido iniciadas durante o retorno elástico (spring back) ou arredondamento (rerounding) da mossa. Quando a mossa se move para fora, maiores tensões de dobramento e deformações são geradas no sulco, as quais podem causar iniciação de trincas, ou fazer com que trincas já existentes possam crescer por meio de rasgamento dútil. Variações das pressões de operação e partidas e paradas dos dutos podem ajudar na iniciação e/ou no crescimento de trincas na raiz dos sulcos.

Como na região do dano mossa e sulco ocorre encruamento do material e consequente elevação da resistência ao escoamento e redução da dutilidade e tenacidade local do material, isto implica que trincas induzidas pela deformação a frio podem se estender mais facilmente nesta região (tenacidade mais baixa) do que no material base fora da mesma (região de tenacidade 


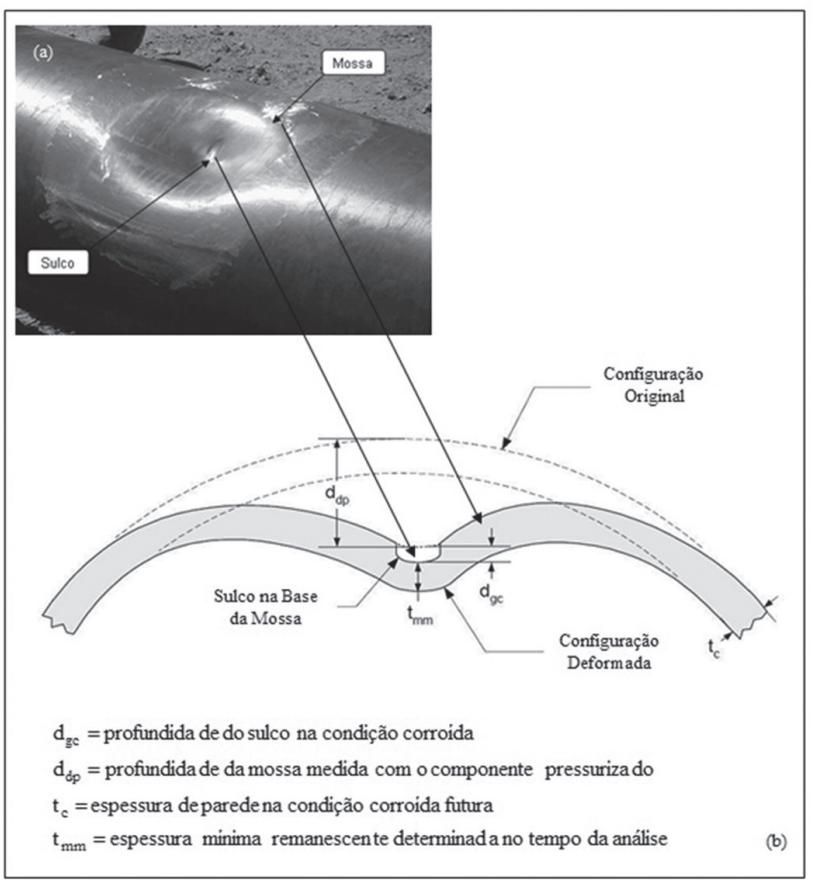

Figura 2 - (a) Foto ilustrativa do dano mossa e sulco [12] e (b) dimensões do dano mossa e sulco e nomenclatura adotada pela referência [8].

mais alta). O comportamento da trinca a partir deste ponto é dependente da espessura remanescente do tubo, da tenacidade à fratura do material base, da geometria do tubo e da trinca, e do nível de tensão nominal. Segundo Mayfield et al. [10], este comportamento poderá ser das seguintes formas: (1) a trinca não se estende mais, (2) a trinca se estende através da parede, mas permanece estável (condição de vazamento), ou (3) a trinca se estende através da parede, tornando-se instável e se propaga (condição de ruptura).

A falha do duto que ocorre imediatamente quando o dano mecânico é introduzido é referida como 'falha imediata', enquanto que a falha que ocorre algum tempo depois que este dano foi introduzido é referido como 'falha retardada'. Uma falha retardada pode ser causada pelo crescimento/desenvolvimento de um mecanismo de dano, tal como fadiga, corrosão sob tensão, plasticidade 'dependente do tempo' ou "cold creep", por um aumento de pressão e/ou por outras formas de carregamento $[10,13]$.

\section{Métodos para Avaliar a Adequação ao Uso de Duto com Dano Mossa e Sulco}

O propósito da 'avaliação de integridade' de duto é assegurar que o mesmo é seguro e protegido. Uma parte importante da avaliação de integridade é a avaliação de 'adequação ao uso' (fitness-for-purpose), também conhecida como 'habilitação para serviço' (fitness-for-service), do duto. A avaliação de 'adequação ao uso' refere-se ao cálculo da condição de falha de um defeito estrutural [14].

Segundo Jones e Hopkins [14], uma avaliação de 'adequação ao uso' de um duto contendo um defeito pode ser estimada por uma variedade de métodos que vão, desde experiências prévias relevantes, modelagem de testes (métodos empíricos), até uma avaliação crítica de engenharia onde a severidade de um defeito é calculada analiticamente.

Diversas publicações técnicas, tais como Alexander e Brownlee [1], Francis et. Al. [3], Seevam et al. [6], Jandu et al. [7], API 579-1/ASME FFS-1 [8], Cosham e Hopkins [9], Cosham [11], Spiekhout et al. [16], Jones e Hopkins [18], Bood et al. [21], Roovers et al. [22], Bai e Song [24], citam e/ou descrevem os métodos analíticos semi-empíricos abaixo relacionados, em ordem cronológica, que visam avaliar a adequação ao uso de duto com dano mossa e sulco. Detalhes gerais sobre cada um destes métodos encontram-se descritos, respectivamente, nos itens 3.1 ao 3.6.

I) Método semi-empírico proposto por Gasunie (1986)

II) Método da fratura mossa e sulco semi-empírico original desenvolvido pela British Gas (1992)

III) Método da fratura mossa e sulco semi-empírico da British Gas modificado (1997)

IV) Método semi-empírico proposto por Bai e Song (1997) V) Métodos adotados pelo API 579-1/ASME FFS-1 (2007)

VI) "Novo" método desenvolvido por Kiefner e Andrew Francis, sob solicitação do PRCI (2008)

\subsection{Método Semi-Empírico Proposto por Gasunie}

Segundo Cosham [11], Gasunie conduziu um número de testes em vasos contendo mossas circunferenciais e sulcos e propôs uma fórmula semi-empírica para predizer a tensão de falha do dano mossa e sulco, baseada no modelo original de Dugdale (strip yield model) modificado por Burdekin e Stone, que é apresentado em Anderson [15].

No desenvolvimento deste método, foram feitas as considerações e simplificações, enumeradas a seguir, relativas à geometria do defeito mossa e sulco [11]:

i) A mossa é contínua e tem uma largura constante;

ii) Um entalhe agudo, com profundidade constante, é localizado no ponto mais profundo da mossa e se estende na direção axial do tubo.

A expressão do método proposto por Gasunie [16] encontrase apresentada em Cosham [11] na forma da Equação 2 a seguir.

$$
\frac{\sigma_{\theta}}{\sigma_{Y}}=\frac{2}{\pi} \cos ^{-1}\left[\exp -\left\{\frac{\pi}{8 \sigma_{Y}^{2} d}\left(\left.1000 \frac{E}{A}\right|_{v}-17.6\right)\left[Y_{1}+6 Y_{2}\left(\frac{D}{t} \frac{H}{D}\right)\right]^{-2}\right\}\right]
$$

Onde:

$$
\begin{aligned}
& Y_{1}=1.12-0.23\left(\frac{d}{t}\right)+10.6\left(\frac{d}{t}\right)^{2}-21.7\left(\frac{d}{t}\right)^{3}+30.4\left(\frac{d}{t}\right)^{4} \\
& Y_{2}=1.12-1.39\left(\frac{d}{t}\right)+7.32\left(\frac{d}{t}\right)^{2}-13.1\left(\frac{d}{t}\right)^{3}+14.0\left(\frac{d}{t}\right)^{4}
\end{aligned}
$$

${ }^{1}$ Como o método desenvolvido por Kiefner e Andrew Francis é referido no artigo técnico de Jandu et al. [7] apenas como o "Novo" modelo, essa mesma denominação foi adotada neste trabalho. 
$\sigma_{\theta} \quad$ tensão circunferencial para falha $\left(\mathrm{N} / \mathrm{mm}^{2}\right)$

$\sigma_{Y} \quad$ resistência ao escoamento $\left(\mathrm{N} / \mathrm{mm}^{2}\right)$

E módulo de Young $\left(207.000 \mathrm{~N} / \mathrm{mm}^{2}\right)$

$C_{v} \quad$ energia de impacto Charpy tipo $\mathrm{V}$ absorvida pelo espécime tamanho $2 / 3$ da espessura $(\mathrm{J})$

A área de fratura para um espécime Charpy $2 / 3$ $\left(53,55 \mathrm{~mm}^{2}\right.$ para um espécime Charpy $\left.2 / 3\right)\left(\mathrm{mm}^{2}\right)$

$d \quad$ profundidade do defeito de perda de metal sulco $(\mathrm{mm})$

$H$ profundidade da mossa medida com o duto sob $t$ pressão, após a remoção do indentador (mm)

$D$ espessura de parede do tubo (mm) diâmetro externo do tubo (mm)

A profundidade da mossa assumida no método é a profundidade da mossa depois do retorno elástico (spring back), medida na condição de pressão. $\mathrm{O}$ relacionamento empírico entre a energia de impacto Charpy $\left(C_{v}\right)$ e o fator intensidade de tensão crítico $\left(K_{c}\right)$, inicialmente desenvolvido por Battelle $\left(K_{c}=\right.$ $C_{v} 10^{3} \mathrm{E} / \mathrm{A}$, expresso em $\mathrm{N} / \mathrm{mm}^{1,5}$ ), foi modificado para obter um razoável ajuste entre os prognósticos do método de Gasunie e os resultados dos testes de ruptura da British Gas em anéis e vasos com mossa e sulco reportados em Jones [17 apud 16].

\subsection{Método da Fratura Mossa e Sulco Semi-Empírico Original Desenvolvido pela British Gas}

O método da fratura semi-empírico para predizer a tensão de falha de uma mossa contendo um sulco foi desenvolvido pela British Gas (BG) em 1981, e descrito, em termos gerais, em Jones e Hopkins [18] e em Hopkins et al. [19]. Uma descrição detalhada deste método, incluindo as suas equações, foi publicada por Hopkins [20]. Este método foi adotado pelo EPRG nas suas recomendações para a avaliação de dano mecânico [21,22].

A aplicação de uma análise estatística, embora sendo uma rota ideal para derivação de simples limites de segurança, pode resultar em considerável conservadorismo para algum tipo de dano. Um método analítico foi então desenvolvido para danos que extrapolam os limites de métodos empíricos (regra mais conservativa), tendo como base a mecânica da fratura elasto-plástica, utilizando o modelo "Bilby-Cottrell-Swinden Dislocation Model" (modificação do "strip yield model" de Dugdale) que inclui o efeito da plasticidade na ponta da trinca, e é expresso por uma função que permite ser aplicada para qualquer geometria de tubo [21].

Este método foi calibrado (ajustado) usando o resultado de testes de ruptura para 111 anéis e 21 vasos de pressão contendo entalhes usinados nas mossas. Primeiro foi produzido o entalhe e, posteriormente, a mossa, ambos feitos com o equipamento a ser testado na condição de despressurizado. O método é definido conforme a Equação 3 a seguir [23].

$$
\begin{aligned}
& \frac{\sigma_{Q}}{\bar{\sigma}}=\frac{2}{\pi} \cos ^{-1}\left[\exp -\left\{113 \frac{1.5 . \mathbb{E}}{\frac{2}{\sigma} A d}\left[Y_{1}\left(1-1.8 \frac{H_{0}}{D}\right)+Y_{2}\left(10.2 \frac{R}{t} \frac{H_{0}}{D}\right)\right]^{-2} \exp \left[\frac{\ln \left(0.738 C_{\nu}\right)-K_{1}}{K_{2}}\right]\right\}\right](3) \\
& \text { Onde: } \\
& \bar{\sigma}=1.15 \sigma_{Y}\left(1-\frac{d}{t}\right) \\
& Y_{1}=1.12-0.23\left(\frac{d}{t}\right)+10.6\left(\frac{d}{t}\right)^{2}-21.7\left(\frac{d}{t}\right)^{3}+30.4\left(\frac{d}{t}\right)^{4} \\
& Y_{2}=1.12-1.39\left(\frac{d}{t}\right)+7.32\left(\frac{d}{t}\right)^{2}-13.1\left(\frac{d}{t}\right)^{3}+14.0\left(\frac{d}{t}\right)^{4} \\
& K_{1}=1.9 \\
& K_{2}=0.57 \\
& H_{0}=1.43 H
\end{aligned}
$$

A nomenclatura para variáveis usadas nas expressões acima é a mesma já apresentada no Item 3.1. Exceção feita às variáveis específicas do método da Bristish Gas, as quais encontram-se definidas a seguir.

$$
\begin{array}{ll}
\mathrm{D} & \begin{array}{l}
\text { diâmetro externo do tubo }(\mathrm{mm}) \\
\text { profundidade da mossa medida com o duto sem }
\end{array} \\
H_{0} & \begin{array}{l}
\text { pressão (mm) } \\
K_{1}
\end{array} \\
\text { parâmetro de regressão não-linear } \\
K_{2} & \text { parâmetro de regressão não-linear } \\
\bar{\sigma} & \text { tensão de colapso plástico }\left(\mathrm{N} / \mathrm{mm}^{2}\right)
\end{array}
$$

As considerações e simplificações relativas à geometria do defeito mossa e sulco, referidas no método de Gasunie, são também aplicáveis a este método da British Gas [11]. Também de forma análoga ao método de Gasunie, o comprimento da mossa e o comprimento do sulco não são considerados na equação do método da British Gas. A relação entre a tenacidade à fratura e a energia de impacto Charpy absorvida pelo espécime tamanho 2/3 de espessura, que é diferente da usada no método de Gasunie, foi determinada por meio de uma análise de regressão não-linear dos dados dos testes dos anéis e vasos de pressão contendo mossa e sulco [23].

\subsection{Método da Fratura Mossa e Sulco Semi-Empírico da British Gas Modificado}

Uma pequena revisão do modelo da fratura mossa e sulco semi-empírico original (descrito no Item 3.2) foi empreendida pela British Gas (BG) e é referida em Francis et al. [27]. A formulação básica do modelo não foi mudada, porém a tensão de escoamento (tensão de colapso) foi modificada para levar em conta o comprimento do sulco, e a correlação da tenacidade com a energia conseguida no ensaio Charpy foi também alterada.

Segundo Cosham [11], as considerações e simplificações 
relativas à geometria do defeito mossa e sulco, descritas no método de Gasunie, são também aplicáveis a este método.

O método da fratura mossa e sulco semi-empírico modificado é expresso pela Equação 4:

$\frac{\sigma_{\theta}}{\bar{\sigma}}=\frac{2}{\pi} \cos ^{-1}\left[\exp -\left\{\frac{\pi}{8 \bar{\sigma}^{2} d}\left(\frac{E C_{v 0}}{A}\left(\frac{C_{v}}{C_{v 0}}\right)^{\frac{1}{\beta}}\right)\left[Y_{1}\left(1-\frac{1.8 H_{o}}{2 R}\right)+Y_{2}\left(10.2 \frac{R}{t} \frac{H_{o}}{2 R}\right)\right]^{-2}\right\}\right]$

Onde:

$\bar{\sigma}=\frac{1.15 \sigma_{Y}\left(1-\frac{d}{t}\right)}{\left(1-\frac{1.8 H_{o}}{2 R}\right)\left(1-\frac{1}{M} \frac{d}{t}\right)} ;$ e $M=\sqrt{1+0.26\left(\frac{2 c}{\sqrt{R t}}\right)^{2}}$

A nomenclatura para variáveis usadas nas expressões acima é a mesma já apresentada no Item 3.1. Exceção feita às variáveis específicas do método da Bristish Gas modificado, as quais encontram-se definidas a seguir.

$\begin{array}{ll}C_{v 0} & \text { parâmetro de regressão não-linear }{ }^{(2)} \\ \beta & \text { parâmetro de regressão não-linear }{ }^{(2)} \\ 2 c & \begin{array}{l}\text { comprimento do defeito de perda de metal - } \\ \text { sulco }(\mathrm{mm})\end{array}\end{array}$

\subsection{Método Semi-Empírico Proposto por Bai e Song}

Uma fórmula semi-empírica para predizer a pressão de ruptura de um tubo contendo uma mossa combinada com entalhe longitudinal foi proposta por Bai e Song. O método foi desenvolvido usando a condição de fratura segundo o modelo "Bilby-Cottrell-Swinden Dislocation Model", mas com modificações para a tenacidade, para a função determinada e para a tensão de colapso [24].

As bases teóricas implícitas para o método de fratura mossa e sulco de Bai e Song são muito similares às do método da British Gas, contudo a tenacidade foi adotada diretamente do método de Gasunie, e o efeito do comprimento do entalhe (sulco) foi considerado na função determinada e na tensão de colapso.

Segundo Bai e Song [24], no desenvolvimento do modelo foram feitas as considerações e simplificações, enumeradas a seguir, relativas à geometria do defeito mossa e sulco:

i) Assume-se que a mossa é contínua e tem uma largura constante;

ii) O fator concentrador de tensão é considerado como sendo um entalhe localizado no ponto mais profundo da mossa, de comprimento infinito e profundidade constante. O entalhe é longitudinal com comprimento $L=2 c$ e profundidade $d$.

A resistência à ruptura de um tubo contendo uma mossa combinada com um sulco longitudinal segundo o método proposto por Bai e Song [24] é dada pela Equação 5.

2 Em Cosham [11] os valores para estes parâmetros não encontram-se especificados.

$$
\frac{\sigma_{\theta}}{\bar{\sigma}}=\frac{2}{\pi} \cos ^{-1}\left[\exp -\left\{\frac{\pi}{8 \bar{\sigma}^{2} d}\left(1000 \frac{E}{A}\left(C_{v}-17.6\right)\right]\left[\frac{F}{\sqrt{Q}}\left(1-\frac{1.8 H_{o}}{2 R}+5.1 H \frac{R}{t} \frac{H_{o}}{2 R}\right)\right]^{-2}\right\}\right]
$$

$$
\begin{aligned}
& \text { Onde: } \\
& \qquad \overline{\tau^{\prime}}=\left\langle\mathrm{Ut}_{Y} \frac{\left(1-\frac{d}{t}\right)}{\left(1-\frac{d}{t} \frac{1}{M}\right)}\right.
\end{aligned}
$$

$$
\begin{array}{ll}
M=\sqrt{1+0.6275\left(\frac{2 c}{\sqrt{D t}}\right)^{2}-0.003375\left(\frac{2 c}{\sqrt{D t}}\right)^{4}} \quad \text { para }\left(\frac{2 c}{\sqrt{D t}}\right)^{2} \leq 50.0 \\
M=0.032\left(\frac{2 c}{\sqrt{D t}}\right)^{2}+3.3 & \text { para }\left(\frac{2 c}{\sqrt{D t}}\right)^{2}>50.0
\end{array}
$$

A nomenclatura para variáveis usadas nas expressões acima é a mesma já apresentada nos itens 3.1 ao 3.3, exceção feita à variável específica do método de Bai e Song, a, definida como sendo um fator de proporcionalidade entre a resistência ao escoamento e a tensão de colapso plástico.

As funções $F, H$ e $Q$ são propostas por Newman e Raju [25 apud 24] e apresentadas, também, em Cosham [11]. O relacionamento empírico entre a energia de impacto Charpy-V e a tenacidade à fratura, desenvolvido por Gasunie, foi usado na equação do método [24].

\subsection{Método Adotado pelo API 579-1/ASME FFS-1}

O padrão API 579-1/ASME FFS-1 [8] adota três níveis de avaliação (1, 2 e 3 ) para o dano tipo mossa e sulco. Quanto menor o nível de avaliação $(1<2<3)$, menor será a quantidade de dados, bem como a exatidão requerida para os mesmos, para que sejam realizados os cálculos pertinentes. Na avaliação nível 1 é usado o método empírico desenvolvido pelo EPRG [28], apenas com pequena modificação na forma da apresentação gráfica. Por sua vez, nas avaliações níveis 2 e 3 é adotado o método da fratura mossa e sulco original desenvolvido pela British Gas (Item 3.2), diferenciando-se deste, basicamente, apenas com relação à nomenclatura para os termos utilizados nas equações e a forma de apresentação das mesmas.

\section{6. "Novo" Método Desenvolvido por Kiefner e Andrew Francis, Sob Solicitação do PRCI}

O desenvolvimento do "novo" método proposto por Kiefner e Andrew Francis [7] iniciou-se por uma revisão dos modelos existentes para avaliação de dano mecânico em dutos. Foi observado que vários aspectos destes modelos não poderiam ser harmonizados com a moderna teoria da mecânica da fratura elasto-plástica. Em virtude disto, foi procurado elaborar um modelo que poderia justificar tanto esta teoria quanto os dados disponíveis encontrados para os testes de ruptura de dutos com dano mecânico [7].

Este "novo" método incorpora os efeitos de tensões induzidas pela pressão, tensões residuais devido à mossa, fator de concentração de tensão de acordo com a presença do sulco e o fator intensificador de tensão de acordo com a presença de trinca. $\mathrm{O}$ mesmo também incorpora um termo para determinar 
a profundidade do trincamento baseado na profundidade da mossa, na largura da banda de escorregamento (slip-band) e no tamanho de grão [7].

Em geral, a falha de uma estrutura de aço contendo defeito planar tipo trinca ocorrerá de acordo com a combinação de fratura e colapso plástico. Em reconhecimento a isto, tal como nos outros métodos mostrados anteriormente, a falha é prevista de ocorrer se $K_{r}$ (parâmetro de fratura, adimensional) satisfaz a Inequação descrita a seguir [7].

$$
K_{r} \geq F\left(L_{r}\right)
$$

Onde a função $F$ define o 'Diagrama de Avaliação de Falha' (FAD - Failure Assessment Diagram) ou 'Linha de Avaliação de Falha' (FAL - Failure Assessment Line). No caso do "novo" método proposto $F$ é dado por:

$$
\begin{aligned}
& F=\left(1+0.5 L_{r}^{2}\right)^{-1 / 2}\left(0.3+0.7 \exp \left(-0.6 L_{r}^{6}\right)\right) \\
& \text { E } K_{r} \text { é dado por: } \\
& K_{r}=\frac{K}{K_{\text {mat }}}+\rho
\end{aligned}
$$

Onde $K$ denota o fator intensidade de tensão, $K_{\text {mat }}$ é a tenacidade à fratura do material e $\rho$ é o fator correção de plasticidade (adimensional). Em unidades do sistema internacional, $K$ e $K_{\text {mat }}$ são expressos em $M P a \cdot m^{1 / 2}$.

O parâmetro de colapso plástico, $L_{r}$, adimensional, é dado por:

$$
L_{r}=\frac{\sigma_{r e f}}{\sigma_{y}}
$$

Onde, $\sigma_{\text {ref }}$ é a tensão de referência (carga aplicada) e $\sigma_{y}$ é a tensão de colapso plástico (resistência do material). Em unidades do sistema internacional, $\sigma_{r e f}$ e $\sigma_{y}$ são expressas em $\mathrm{MPa}$. Fazendo um correlação com a Equação 3, tem-se que $\sigma_{r e f}=\sigma_{\theta}$ e $\sigma_{y}=\bar{\sigma}$.

Todas as subequações que definem os parâmetros $K, K_{\text {mat }}, \sigma_{\text {ref }}$ e $\sigma_{y}$ encontram-se no artigo de Jandu et al. [7].

\section{Análise Técnica dos Métodos Existentes para Avaliar a Adequação ao Uso de Dutos com Dano Mossa e Sulco}

Uma análise técnica dos métodos descritos nos itens 3.1 ao 3.6 foi realizada com o objetivo de determinar qual seria o 'mais recomendado' para avaliar a adequação ao uso (fitness-forpurpose) de dutos onshore com dano mossa e sulco. Os métodos foram analisados, basicamente, quanto aos seguintes aspectos:

- Fundamentação teórica;

- Natureza e disponibilidade dos dados requeridos;

- Exatidão dos resultados (validação experimental).

Os resultados das análises realizadas para cada um dos aspectos acima citados encontram-se apresentados nos itens 4.1 ao 4.3 a seguir.

\subsection{Fundamentação Teórica}

Todos os métodos analíticos disponíveis para avaliar a adequação ao uso de dutos com dano mossa e sulco, descritos nos itens 3.1 ao 3.6, baseiam-se nos princípios da mecânica da fratura clássica, que leva em consideração que a falha dependerá tanto da resistência do material ao colapso plástico (governada pela tensão de colapso - "flow stress") quanto da resistência à fratura (governada pela tenacidade à fratura do material $-K_{I C}$ ou $K_{\text {mat }}$ ). Vale informar, também, que todos estes métodos podem ser apresentados graficamente usando o 'Diagrama de Avaliação de Falha' (FAD).

Com relação às diferenças entre os métodos analisados, uma das mais notáveis diz respeito à definição da composição da tensão de colapso plástico e do parâmetro tenacidade à fratura do material (relação entre $K_{I C}$ e $C_{v}$ ). Adicionalmente, existem também variações com relação às composições da tensão de membrana primária e da tensão de flexão secundária, e consideração ou não, nos cálculos, da tensão de membrana secundária e dos parâmetros que definem o dano mossa e sulco, tais como a profundidade do sulco e da mossa e o comprimento do sulco.

Sumariamente, encontra-se descrito em Cosham [11], que todos os métodos semi-empíricos analisados dentro do projeto PDAM (Pipeline Defect Assessment Manual), apresentados nos itens 3.1 ao 3.5, são pequenas variações do método da fratura mossa e sulco originalmente desenvolvido pela British Gas, que o método de Gasunie é fundamentalmente falho e que os métodos BG modificado e de Bai e Song continham vários parâmetros empíricos que até então não tinham sido publicados.

Referente ao "novo" método (não avaliado no projeto PDAM), o mesmo acrescenta algumas das limitações do método da fratura mossa e sulco original da British Gas por explicitamente levar em consideração o efeito de micro-trinca e tensão residual (presença de tensão de membrana secundária) conhecida de ocorrer na região do sulco. Parâmetros de ajustes adicionais foram determinados iterativamente associando valores para cada parâmetro e visualmente observando-se a concordância dos dados com o 'Diagrama de Avaliação de Falha' [6].

\subsection{Natureza e Disponibilidade dos Dados Requeridos}

Com relação aos dados necessários aos cálculos inerentes aos métodos analíticos semi-empíricos analisados, os mesmos poderão, normalmente, ser conseguidos sem maiores dificuldades, exceção feita à 'profundidade da mossa', que é considerada nos cálculos como sendo na pressão zero, e à 'energia do impacto Charpy'.

No caso da 'profundidade da mossa', considerando que a mesma pode ser coletada (medida) com o duto pressurizado, se faz necessário adotar um fator de correção para o arredondamento (rerounding) do tubo, sendo que alguns dos métodos analisados já sugerem este fator de correção, porém, outros não.

Com relação a 'energia de impacto Charpy', caso não se tenha esta informação especificamente para o tubo do duto com dano mossa e sulco a ser analisado, sugere-se que seja adotado o valor disponível na literatura técnica para a especificação do 
material correspondente a este tubo.

Adicionalmente, ressalta-se que será necessária a medição, em campo, da profundidade da mossa e do sulco, quando utilizando qualquer um dos métodos analisados, e, adicionalmente, o comprimento do sulco, para os métodos de Bai e Song e "novo" método. Visando uma boa exatidão para os valores destes parâmetros, considera-se que os mesmos terão que ser medidos por meio de técnicas de inspeção aplicadas externamente ao duto.

De forma mais particular, tem-se necessidade de realização de uma análise de tensões da forma numérica para a avaliação nível 3 do API 579-1/ASME FFS-1 e para o "novo" método. Ainda com relação ao "novo" método, a determinação prévia de alguns parâmetros adicionais, tais como a profundidade de micro-trinca, tamanho de grão e largura da banda de escorregamento (slipband), se faz necessária aos cálculos realizados pelo mesmo. Ressalta-se que a determinação destes parâmetros, especificamente para o material do tubo do duto com dano mossa e sulco a ser analisado, normalmente será muito difícil. $\mathrm{O}$ "novo" método então sugere a utilização destes parâmetros conforme dados disponíveis na literatura técnica para material similar ao do tubo do duto em análise. Isto retira qualquer possibilidade de julgamento da exatidão da resposta deste método.

\subsection{Exatidão dos Resultados (Validação Experimental)}

A exatidão dos resultados referentes aos métodos BG original, BG modificado e Gasunie foi verificada diretamente por este trabalho técnico por meio de validação experimental (análise estatística). Foi feito um estudo comparativo entre as tensões 'calculadas pelos métodos' (y) com a tensões 'reais' (x) causadas pelas pressões de falha para 67 testes de ruptura realizados no projeto PDAM [11], cujos resultados encontramse plotados no gráfico mostrado na Figura 3.

No gráfico da Figura 3 pode ser observado que, para todos os métodos analisados, existe um grande espalhamento das tensões calculadas versus tensões reais medidas, as inclinações das retas (funções) geradas por meio de análise de regressão linear são similares, e que todos são não conservativos para tensões de falha reais até $200 \mathrm{MPa}$ e conservativos para tensões de falha reais superiores a este valor.

Adicionalmente, foi realizada uma análise de variância para os métodos BG original, BG modificado e Gasunie, com resultado demonstrando que os mesmos são estatisticamente diferentes, e também executada uma análise estatística descritiva cujos resultados encontram-se apresentados na Tabela 1.

Tabela 1 - Resultados da análise estatística descritiva executada para os métodos BG original, BG modificado e Gasunie

\begin{tabular}{|l|c|c|c|c|}
\hline \multirow{2}{*}{ Método } & \multicolumn{4}{|c|}{$\begin{array}{r}\text { Resultados da análise estatística considerando 67 } \\
\text { testes realizados no projeto PDAM }\end{array}$} \\
\cline { 2 - 5 } & $\begin{array}{c}\text { Valor } \\
\text { médio } \\
\text { de }(\mathrm{y} / \mathrm{x})\end{array}$ & $\begin{array}{c}\text { Desvio } \\
\text { padrão } \\
\text { de }(\mathrm{y} / \mathrm{x})\end{array}$ & $\begin{array}{c}\text { Coeficiente } \\
\text { de variação } \\
\text { de }(\mathrm{y} / \mathrm{x})\end{array}$ & $\begin{array}{c}\text { Coeficiente } \\
\text { de regressão } \\
\text { de }(\mathrm{y} / \mathrm{x})\end{array}$ \\
\hline BG original & 1,00 & 0,61 & 0,61 & 0,68 \\
\hline BG modificado & 1,68 & 0,78 & 0,47 & 0,69 \\
\hline Gasunie & 1,66 & 0,74 & 0,45 & 0,69 \\
\hline
\end{tabular}

Nota (1): Estes 67 testes referem-se aos testes realizados que dispunham de todos os dados necessários às equações dos métodos analisados, exclusos os casos em que o sulco gerado no tubo de teste foi esmerilhado.

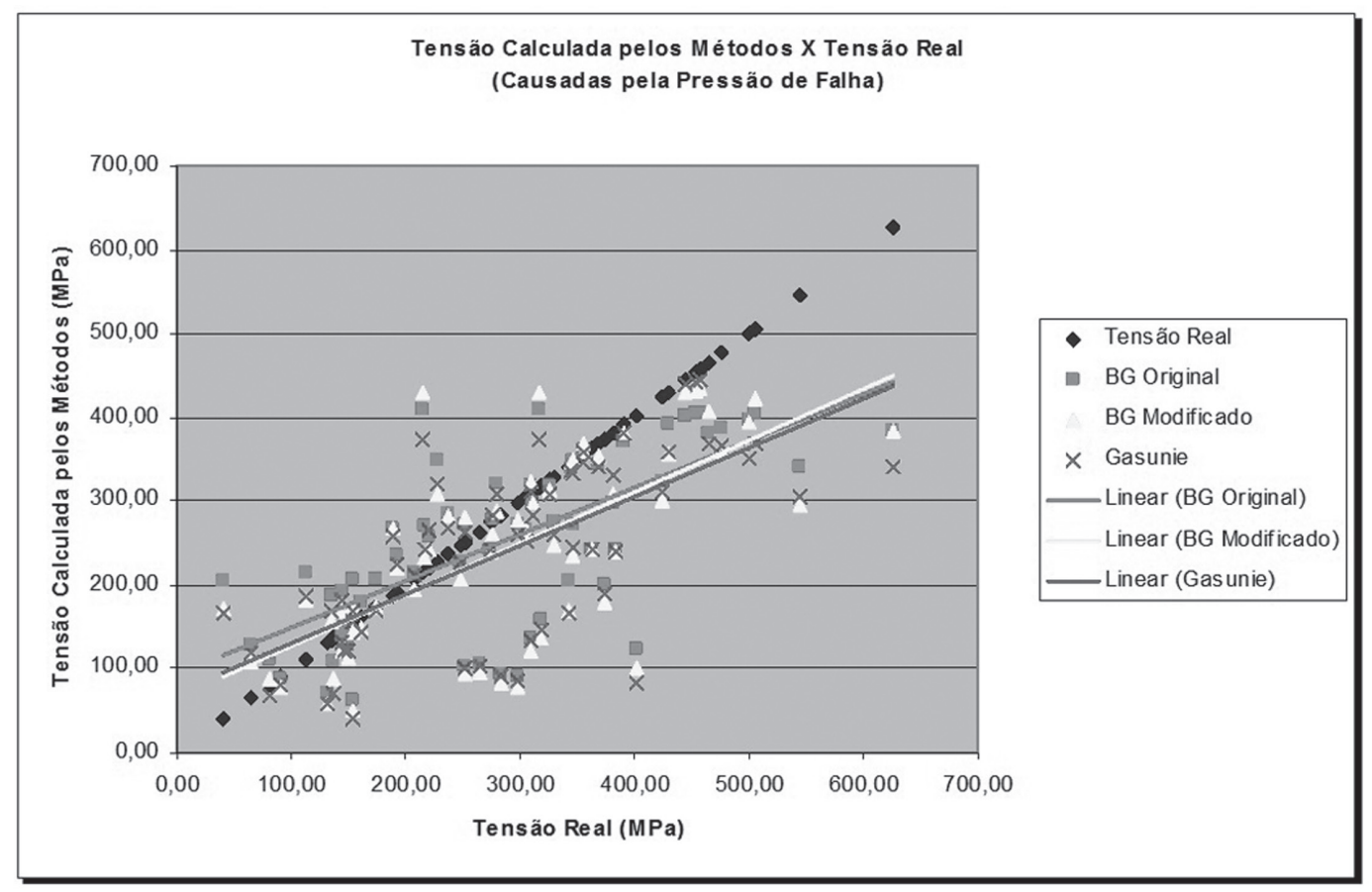

Figura 3 - Tensões calculadas pelos métodos BG original, BG modificado e Gasunie versus tensões reais causadas pelas pressões de falha para 67 testes realizados no projeto PDAM. 
Na Tabela 1 observa-se que os valores do coeficiente de regressão são muitos próximos, não sendo este, portanto, um parâmetro que poderia servir como indicativo da diferença de exatidão para os métodos analisados. Entretanto, como o método $\mathrm{BG}$ original apresentou-se com valor médio de " $\mathrm{y} / \mathrm{x}$ " igual a $1 \mathrm{e}$ com menor desvio padrão (menor dispersão sobre a média) entre os métodos analisados, pode-se inferir que o mesmo seria o mais exato em prever a tensão causada pela pressão de falha de duto com dano mossa e sulco, apesar de apresentar um coeficiente de variação maior do que os métodos BG modificado e de Gasunie.

A verificação da exatidão dos resultados para o "novo" método está conforme análise estatística feita por Seevam et al. [6]. O "novo" método e o método da fratura mossa e sulco original da British Gas foram comparados usando, também, dados de testes realizados no projeto PDAM (inclusos os 67 testes referidos na Tabela 2). As pressões de falha reais dos dados dos testes experimentais foram plotadas contra as pressões de falha calculadas por cada método e uma análise de regressão linear foi executada para mensurar a exatidão destes métodos, cujo resultado encontra-se apresentado na Figura 4.

$\mathrm{Na}$ Figura 4, pode ser observado que, para qualquer condição, o método da fratura mossa e sulco original da British Gas apresenta o valor de $\mathrm{R}^{2}$ superior ao do "novo" método, mostrando, estatisticamente, que o método da BG é mais exato do que o "novo" método em predizer a pressão de falha de um duto contendo o dano mossa e sulco.

Como o método de Bai e Song contem vários parâmetros empíricos e que até a publicação do relatório do projeto PDAM os mesmos não tinham sido publicados, não foi então possível a realização de validação experimental (análise estatística) para o mesmo.

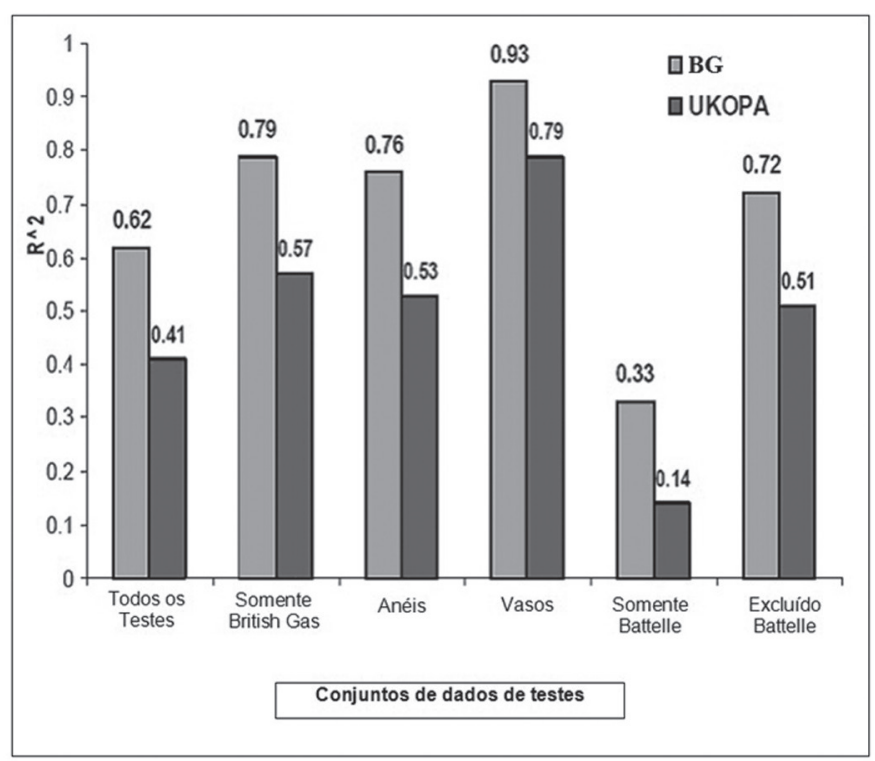

Figura 4 - Comparação do parâmetro $\mathrm{R}^{2}$ (medida do ajuste) para o "novo" método (UKOPA) e o método da fratura mossa e sulco original da British Gas (BG) usando vários conjuntos de dados de testes [6].

\section{Conclusões}

É naturalmente reconhecido na literatura técnica disponível que a pressão de falha de um duto contendo o dano mossa e sulco, sujeito a carregamento de pressão interna, depende da geometria do tubo, dimensões do dano e resistência do material.

A falha do dano mossa e sulco envolve altas deformações plásticas, afinamento da parede do tubo, movimentação da mossa, iniciação de trinca, ruptura dútil e escoamento plástico. Adicionalmente, a base do sulco pode conter trincamento e as propriedades do material na região da mossa e do sulco podem ser adversamente afetadas [6].

De posse da literatura consultada evidencia-se a complexidade em se criar um método para predizer a pressão de falha para o dano 'mossa e sulco' que consiga englobar todos os parâmetros envolvidos na falha deste dano, e que apresente resultados que sejam exatos (calculados sendo, aproximadamente, iguais aos reais medidos).

Com base na análise técnica feita para os diferentes métodos, descrita no Item 4, conclui-se que:

- Nenhum dos métodos atualmente disponíveis para avaliar a adequação ao uso (fitness-for-purpose) de duto com dano mossa e sulco, descritos nos itens 3.1 ao 3.6, de fato contabiliza todos os aspectos do comportamento da falha de um duto com este tipo de dano.

- As limitações principais dos correntes métodos são que eles não efetivamente consideram a regra do crescimento de trinca dútil, os efeitos das mudanças das propriedades locais do material devido à deformação plástica durante a formação da mossa, e o efeito da mudança da profundidade da mossa devido ao retorno elástico (spring back) ou arredondamento (rerounding).

- Todos os métodos analisados são pequenas variações do método da fratura mossa e sulco, originalmente desenvolvido pela British Gas, e todos eles baseiam-se nos princípios da mecânica da fratura clássica, que leva em consideração que a falha dependerá tanto da resistência do material ao colapso plástico (governada pela tensão de colapso - "flow stress") quanto da resistência à fratura (governada pela tenacidade à fratura do material $-K_{I C}$ ou $K_{\text {mat }}$ ).

- Os resultados das validações experimentais (análises estatísticas) realizadas demonstram que o método da fratura mossa e sulco original da British Gas é o 'melhor' método em termos de qualidade de ajuste com os dados dos testes de pressão realizados em anéis e vasos contendo o dano mossa e sulco executados no projeto PDAM.

Por fim, além dos resultados das validações experimentais realizadas, muitos artigos e relatórios técnicos, tais como Francis et al. [26], Roovers et al. [22], Cosham e Hopkins [27] e Macdonald e Cosham [23], referenciam o método da fratura mossa e sulco original desenvolvido pela British Gas como sendo o 'mais recomendado' para avaliar a adequação ao uso de duto com dano mossa e sulco, sendo, portanto, esta conclusão final também endossada por este trabalho técnico. 


\section{Referências Bibliográficas}

[1] ALEXANDER, C.; BROWNLEE, K. Methodology for Assessing the Effects of Plain Dents, Wrinkle Bends and Mechanical Damage on Pipeline Integrity. NACE2007-07139. NACE International Corrosion 2007 Conference \& Expo. Nashville, Tennessee. March 11-15, 2007.

[2] DNV. Process Equipment Failure Frequencies. Technical Note 14 Rev. 1. December, 2000.

[3] FRANCIS, A.; GARDINER, M.; McCALlUM, M. Life Extension of a High Pressure Transmission Pipeline Using Structural Reliability Analysis (IPC02-27159). In: $4^{\text {th }}$ INTERNACIONAL PIPELINE CONFERENCE. Calgary, Canada. September 29 - October 3, 2002.

[4] CONCAWE. Performance of European Cross-Country Oil Pipelines. Statistical Summary of Reported Spillages in 2005 and Since 1971. Report 04/07. May, 2007.

[5] EGIG. $7^{\text {th }}$ Report of the European Gas Pipeline Incident Data Group. $7^{\text {th }}$ EGIG Report 1970-2007. December, 2008.

[6] SEEVAM, P. et al. Modelling of Dent and Gouges, and the Effect on the Failure Probability of Pipelines (IPC2008-64061). In: $7^{\text {th }}$ INTERNACIONAL PIPELINE CONFERENCE. Calgary, Canada. September 29 - October 3, 2008.

[7] JANDU, C. et al. Towards a New Limit State Function for Determining the Failure Pressure of a Pipeline Containing Mechanical Damage. IPC2008-64304. In: $7^{\text {th }}$ International Pipeline Conference. Calgary, Canada. September 29 - October 3, 2008.

[8] API 579-1/ASME FFS-1. Fitness-For-Service. API 579 Second Edition. June 2007. Part 12.

[9] COSHAM, A.; HOPKINS, P. The Pipeline Defect Assessment Manual (PDAM) - A Report to the PDAM Joint Industry Project. Newcastle, UK. May 2003. Chapter 25.

[10] MAYFIELD, M. E.; MAXEY, W. A.; WILKOWSKI, G. M. Fracture Initiation Tolerance of Line Pipe. Paper F, 6th Symposium on Line Pipe Research, American Gas Association, Houston, Texas, 1979.

[11] COSHAM, A. Assessment Methods for Dents and Gouges in Pipelines. A Report to The Pipeline Defect Assessment Manual (PDAM) - Joint Industry Project. February 2001.

[12] EA Services. Composite Wrap Services - Dented Repairs. Disponível em: <http://www.easervices.com/composite-wrap. htm\#dented $>$. Acesso em: 16/03/2009.

[13] COSHAM, A.; HASWELL, J.; JACKSON, N. Reduction Factors for Estimating the Probability of Failure of Mechanical Damage Due to External Interference. IPC2008-64345. In: $7^{\text {th }}$ International Pipeline Conference. Calgary, Canada. September 29 - October 3, 2008.

[14] JONES, R. P.; HOPKINS, P. Managing ILI Projects to Get the Results You Need. Pigging Products and Services Association. 2005.

[15] ANDERSON, T. L. Fracture Mechanics - Fundamentals and Applications. CRC Press. Taylor \& Francis Group. Third Edition. 2005. Chapters 1, 2, 3, 5 and 9.

[16] SPIEKHOUT, J.; GRESNIGT, A. M.; KONING, C.; WILDSCHUT, H. Calculation Models for the Evaluation of the Resistance Against Mechanical Damage of Pipelines. 3R
International, 25. Jahrgang, Heft, pp 198-203, 4 April 1986. [17] JONES, D. G. The Significance of Mechanical Damage in Pipelines. 3R International, 21, Jahrgang, Heft. 7 July, 1982.

[18] JONES, D. G.; HOPKINS, P. The Influence of Mechanical Damage on Transmission Pipeline Integrity. Paper A21. International Gas Research Conference. London, UK. 13-16 June, 1983.

[19] HOPKINS, P.; JONES, D. G.; CLYNE, A. C. The Significance of Dents and Defects in Transmission Pipelines. Paper C376/049. Proceedings International Conference on Pipework, Engineering and Operation. IMechE. London. February, 1989.

[20] HOPKINS, P. The Application of Fitness for Purpose Methods to Defects Detected in Offshore Transmission Pipelines. Conference on Welding and Weld Performance in the Process Industry. London, UK. 27-28 April, 1992.

[21] BOOD, R. et al. EPRG Methods for Assessing the Tolerance and Resistance of Pipelines to External Damage (Part 1). 3R International, 10-11/1999, pp. 739-744.

[22] ROOVERS, P. et al. EPRG Methods for Assessing the Tolerance and Resistance of Pipelines to External Damage. Pipeline Technology Proceedings of the Third International Pipeline Technology Conference. Brugge, Belgium. Vol. II. Elsevier p. 405-25. 2000.

[23] MACDONALD, K. A.; COSHAM, A. Best Practice for the Assessment of Defects in Pipelines - Gouges and Dents. Engineering Failure Analysis 12 (2005) 720-745.

[24] BAI, Y.; SONG, R. Fracture Assessment of Dented Pipes with Cracks and Reliability-Based Calibration of Safety Factor. International Journal of Pressure Vessels and Piping. Vol. 74. pp. 221-229. 1997.

[25] NEWMAN, J. C.; RAJU, I. S. An Empirical Stress-Intensity Factor Equation for the Surface Crack. Engineering Fracture Mechanics, Nos. 1/2, Vol. 15. November 1981, pp. 85-191.

[26] FRANCIS, A. et al. Uprating an In-Service Pipeline Using Reliability-Based Limit State Methods. Conference on Risk Based and Limit State Design and Operation of Pipeline. IBC. Aberdeen, UK. 21-22 May, 1997.

[27] COSHAM, A.; HOPKINS, P. The Effect of Dents in Pipelines - Guidance in the Pipeline Defect Assessment Manual. International Journal of Pressure Vessels and Piping 81 (127139). 2004.

[28] ROOVERS, P. et al. EPRG Methods for Assessing the Tolerance and Resistance of Pipelines to External Damage. Pipeline Technology Proceedings of the Third International Pipeline Technology Conference. Brugge, Belgium. Vol. II. Elsevier p. 405-25. 2000. 